\title{
The Hirota equation over finite fields. Algebro-geometric approach and multisoliton solutions
}

\author{
A. Doliwa ${ }^{\#}$, M. Białecki ${ }^{\dagger \ddagger}$, P. Klimczewski* \\ \# Wydział Matematyki i Informatyki, Uniwersytet Warminsko-Mazurski \\ ul. Żotnierska 14A, 10-561 Olsztyn, Poland \\ *Instytut Fizyki Teoretycznej, Uniwersytet Warszawski \\ ul. Hoża 69, 00-681 Warszawa, Poland \\ ${ }^{\dagger}$ Instytut Geofizyki PAN \\ ul. Księcia Janusza 64, 01-452 Warszawa, Poland \\ ${ }^{\ddagger}$ Instytut Fizyki Teoretycznej, Uniwersytet w Białymstoku \\ ul. Lipowa 41, 15-424 Biatystok, Poland
}

\begin{abstract}
We consider the Hirota equation (the discrete analog of the generalized Toda system) over a finite field. We present the general algebro-geometric method of construction of solutions of the equation. As an example we construct analogs of the multisoliton solutions for which the wave functions and the $\tau$-function can be found using rational functions. Within the class of multisoliton solutions we isolate generalized breather-type solutions which have no direct counterparts in the complex field case.
\end{abstract}

PACS 2003 numbers: 02.30.Ik, 02.10.-v, 05.45.-a

\section{Introduction}

Cellular automata are mathematical models of physical systems in which space and time variables are discrete, and physical quantities take only finite number of values [34]. In spite of simple formulation they are capable to describe wide variety of phenomena, for example traffic flow, immune systems, flow through porous media, fluid dynamics, ferromagnetism. Due to their completely discrete nature cellular automata are naturally suitable for computer simulations. However in this field there are not so many exact analytical results providing solutions with a given global behavior. The goal of this paper is to present a general method of construction of solutions to the cellular automaton associated with the Hirota equation.

The Hirota equation [18], the integrable discretization of the generalized Toda system [27], is one of the most important soliton equations. Its various limits give rise to variety of integrable equations, moreover it is the basic system for studying solvable quantum models [21]. 
Like many other integrable systems the Hirota equation has simple geometric interpretation. In the paper we will use the following (geometric) form of the Hirota equation

$$
\begin{aligned}
& \tau_{m}\left(n_{1}, n_{2}\right) \tau_{m}\left(n_{1}+1, n_{2}+1\right)= \\
& \tau_{m}\left(n_{1}+1, n_{2}\right) \tau_{m}\left(n_{1}, n_{2}+1\right)-\tau_{m-1}\left(n_{1}+1, n_{2}\right) \tau_{m+1}\left(n_{1}, n_{2}+1\right) .
\end{aligned}
$$

One can describe it as the equation governing the so called Laplace sequence of two dimensional lattices of planar quadrilaterals [7, 10]. This interpretation can be embedded into a more general theory of multidimensional lattices of planar quadrilaterals and their transformations [13, 15, 14, 12]. It was noticed in [8, 9] that geometric constructions in the integrable discrete geometry (in particular, those leading to the Hirota equation) should work also on the level of finite geometries [19, i.e., geometries over finite fields [24]. This observation has been developed in the present paper.

The question of construction of integrable systems with solutions taking values in a discrete set (soliton cellular automata) is not new and it was undertaken in a number of papers, see for example [5, 6, 33, 28]. In particular, in paper |5 other Hirota equation (equivalent to the discrete sine-Gordon equation [17]) is investigated in the context of finite fields.

In the paper we present general method of finding solutions of the Hirota equation (11) over finite fields by using algebro-geometric methods, standard in a complex domain in the soliton theory 222 , 3. We change however the field of definition of the underlying algebraic curves from the complex numbers to a finite field (see also earlier algebro-geometric papers [16, 29, 2, 32] where such a possibility was considered).

It turns out that algebraic geometry over finite fields has become recently very important in practical use, especially in modern approaches to public key cryptography [20] and in the theory of error correcting codes [31]. With respect to the last application we would like to mention paper [30] where dynamics of the finite Toda molecule (a reduction of the Hirota equation) over finite fields was studied from the point of view of a decoding algorithm.

We do not present here direct connection of the objects of the paper with the integrable discrete geometry over finite fields. This connection becomes clear when approach to the Hirota equation presented here (see also [22]) is compared with results of [1, 11, 12], where methods of algebraic geometry over the field of complex numbers have been applied to construct integrable geometric lattices.

The layout of the paper is as follows. In Section 2 we present the general algebrogeometric scheme for construction of solutions of the Hirota equation. Section 3 is devoted to construction of multisoliton solution on an algebraic curve starting from the vacuum solution. Finally, in Section 1 we give in explicit form solutions of the Hirota equation for the background algebraic curve being the projective line. In particular, we present the mechanism (based on the action of the Galois group) of generation of generalized breather-type solutions and we discuss periodicity of the solutions. 


\section{Solutions of the Hirota equation from algebraic curves over finite fields}

This Section is motivated by algebro-geometric (over the complex field) approach to the Hirota equation (in a different form) [22 and by papers [1, 11, 12 on application of algebro-geometric methods to integrable discrete geometry. It turns out that basic ideas of the algebro-geometric approach to soliton theory can be transferred to the level of integrable systems in finite fields. The notions and results form the theory of algebraic curves over finite fields which we use here can be found in 31.

Consider an algebraic projective curve, absolutely irreducible, nonsingular, of genus $g$, defined over the finite field $\mathbb{K}=\mathbb{F}_{q}$ with $q$ elements, where $q$ is a power of a prime integer $p$. By $\mathcal{C}_{\mathbb{K}}$ we denote the set of $\mathbb{K}$-rational points of the curve. By $\overline{\mathbb{K}}$ denote the algebraic closure of $\mathbb{K}$, i.e., $\overline{\mathbb{K}}=\bigcup_{\ell=1}^{\infty} \mathbb{F}_{q^{\ell}}$, and by $\mathcal{C}_{\overline{\mathbb{K}}}$ denote the corresponding (infinite) set of $\overline{\mathbb{K}}$-rational points of the curve. The action of the Galois group $G(\overline{\mathbb{K}} / \mathbb{K})$ (of automorphisms of $\overline{\mathbb{K}}$ which are identity on $\mathbb{K}$ ) extends naturally to action on $\mathcal{C}_{\overline{\mathbb{K}}}$.

Let us choose:

1. two pairs of points $a_{i}, b_{i} \in \mathcal{C}_{\mathbb{K}}, i=1,2$,

2. $N$ points $c_{\alpha} \in \mathcal{C}_{\overline{\mathbb{K}}}, \alpha=1, \ldots, N$, which satisfy the following $\mathbb{K}$-rationality condition

$$
\forall \sigma \in G(\overline{\mathbb{K}} / \mathbb{K}), \quad \sigma\left(c_{\alpha}\right)=c_{\alpha^{\prime}},
$$

3. $\quad N$ pairs of points $d_{\beta}, e_{\beta} \in \mathcal{C}_{\overline{\mathbb{K}}}, \beta=1, \ldots, N$, which satisfy the following $\mathbb{K}$ rationality condition

$$
\forall \sigma \in G(\overline{\mathbb{K}} / \mathbb{K}): \quad \sigma\left(\left\{d_{\beta}, e_{\beta}\right\}\right)=\left\{d_{\beta^{\prime}}, e_{\beta^{\prime}}\right\}
$$

4. $g$ points $f_{\gamma} \in \mathcal{C}_{\overline{\mathbb{K}}}, \gamma=1, \ldots, g$, which satisfy the following $\mathbb{K}$-rationality condition

$$
\forall \sigma \in G(\overline{\mathbb{K}} / \mathbb{K}), \quad \sigma\left(f_{\gamma}\right)=f_{\gamma^{\prime}},
$$

5. the infinity point $h_{\infty} \in \mathcal{C}_{\mathbb{K}}$.

Remark. We consider here only the generic case and assume that all the points used in the construction are generic and distinct. In particular, genericity assumption implies that the divisor $D=\sum_{\gamma=1}^{g} f_{\gamma}$ is non-special.

Remark. It is enough the check the $\mathbb{K}$-rationality conditions in any extension field $\mathbb{L} \supset \mathbb{K}$ of rationality of all the points used in the construction.

Definition 1. Fix $\mathbb{K}$-rational local parameters $t_{i}$ at $b_{i}, i=1,2$. For any integers $n_{1}, n_{2}, m \in \mathbb{Z}$ define the wave function $\psi_{1, m}\left(n_{1}, n_{2}\right)$ as a rational function with the following properties

1. it has pole of the order at most $n_{1}+m+1$ at $b_{1}$ and the pole of order at most $n_{2}-m$ at $b_{2}$ 
2. its first nontrivial coefficient of the expansion in $t_{1}$ at $b_{1}$ is normalized to one,

3 . it has zeros of order at least $n_{1}$ at $a_{1}$, and of order at least $n_{2}$ at $a_{2}$,

4. it has at most simple poles at points $c_{\alpha}, \alpha=1, \ldots, N$,

5 . it has zero at least of the first order at the infinity point $h_{\infty}$,

6. it has at most simple poles at points $f_{\gamma}, \gamma=1, \ldots, g$,

7. it satisfies $N$ constraints

$$
\psi_{1, m}\left(n_{1}, n_{2}\right)\left(d_{\beta}\right)=\psi_{1, m}\left(n_{1}, n_{2}\right)\left(e_{\beta}\right), \quad \beta=1, \ldots, N
$$

For the same set of points we define the wave function $\psi_{2, m}\left(n_{1}, n_{2}\right)$ as a rational function which differs from the function $\psi_{1, m}\left(n_{1}, n_{2}\right)$ only in the properties 1 and 2 $1_{2}$. it has pole of the order at most $n_{1}+m$ at $b_{1}$ and it has pole of the order at most $n_{2}-m+1$ at $b_{2}$,

$2_{2}$. its first nontrivial coefficient of the expansion in $t_{2}$ at $b_{2}$ is normalized to one.

Remark. The functions $\psi_{i, m}\left(n_{1}, n_{2}\right)$ are $\mathbb{K}$-rational, which follows from $\mathbb{K}$-rationality conditions of sets of points in their definition.

Remark. As usual, zero (pole) of a negative order means pole (zero) of the corresponding positive order. Correspondingly one should exchange the expressions "at most" and "at least" in front of the orders of poles and zeros.

Proposition 1. The wave functions $\psi_{i, m}\left(n_{1}, n_{2}\right)$ are unique.

Proof. We show this for the first function. By the Riemann-Roch theorem the dimension (over $\mathbb{K}$ ) of the divisor

$$
\sum_{\alpha=1}^{N} c_{\alpha}+\sum_{\gamma=1}^{g} f_{\gamma}-h_{\infty}-n_{1} a_{1}-n_{2} a_{2}+\left(n_{1}+1+m\right) b_{1}+\left(n_{2}-m\right) b_{2}
$$

is equal to $N+1$. Under the genericity assumption the $N$ constraints (3) and the normalization condition at $b_{1}$ remove the freedom.

Corollary 2. In the next section we show, starting from the wave functions for $N=0$ one can construct the functions for arbitrary $N$.

Remark. To make subsequent formulas more transparent from now on we will skip frequently the dependence on the parameters $n_{1}, n_{2}$.

In the generic case, which we assume in the sequel, when the order of the pole of $\psi_{1, m}$ at $b_{1}$ is $n_{1}+m+1$ and the order of the pole of $\psi_{2, m}$ at $b_{2}$ is $n_{1}-m+1$ denote by $Q_{12, m}\left(n_{1}, n_{2}\right)$ the first nontrivial coefficient of expansion of $\psi_{1, m}$ at $b_{2}$, and by $Q_{21, m}\left(n_{1}, n_{2}\right)$ the first nontrivial coefficient of expansion of $\psi_{2, m}$ at $b_{1}$, i.e.,

$$
\psi_{1, m}=\frac{1}{t_{2}^{n_{2}-m}}\left(Q_{12, m}+\ldots\right), \quad \psi_{2, m}=\frac{1}{t_{1}^{n_{1}+m}}\left(Q_{21, m}+\ldots\right) .
$$


Remark. The functions $Q_{12, m}$ and $Q_{21, m}$ take values in the field $\mathbb{K}$ of the definition of the curve.

Denote by $T_{i}$ the operator of translation in the variable $n_{i}, i=1,2$, for example $T_{1} \psi_{2, m}\left(n_{1}, n_{2}\right)=\psi_{2, m}\left(n_{1}+1, n_{2}\right)$.

Proposition 3. The function $\psi_{1, m}$ satisfies equations

$$
\begin{aligned}
T_{2} \psi_{1, m}-\psi_{1, m} & =\left(T_{2} Q_{12, m}\right) \psi_{2, m} \\
\psi_{1, m+1}-T_{1} \psi_{1, m} & =-\frac{T_{1} Q_{12, m}}{Q_{12, m}} \psi_{1, m} \\
\psi_{1, m-1} & =\frac{1}{Q_{21, m}} \psi_{2, m} .
\end{aligned}
$$

The analogous system for $\psi_{2, m}$ is obtained by exchanging indices 1 with 2 and reversing the shift in the discrete variable $m$

$$
\begin{aligned}
T_{1} \psi_{2, m}-\psi_{2, m} & =\left(T_{1} Q_{21, m}\right) \psi_{1, m} \\
\psi_{2, m-1}-T_{2} \psi_{2, m} & =-\frac{T_{2} Q_{21, m}}{Q_{21, m}} \psi_{2, m} \\
\psi_{2, m+1} & =\frac{1}{Q_{12, m}} \psi_{1, m}
\end{aligned}
$$

Proof. To prove the first equation (4) notice that the left hand side has all properties of the function $\psi_{2, m}$ except of the normalization and must be therefore proportional to $\psi_{2, m}$. The coefficient of proportionality can be fixed comparing expansions at $b_{2}$. Other equation can be proved in the same way.

Fix $\mathbb{K}$-rational local parameters $\tilde{t}_{i}$ at $a_{i}, i=1,2$. In the generic case when the order of $\psi_{i, m}$ at $a_{i}$ is $n_{i}$ by $\rho_{i, m}\left(n_{1}, n_{2}\right)$ denote first non-trivial coefficients of the expansion of $\psi_{i, m}$ at $a_{i}$, i.e.,

$$
\psi_{i, m}=\tilde{t}_{i}^{n_{i}}\left(\rho_{i, m}+\ldots\right)
$$

Similarly, in the generic case when the order of $\psi_{i, m}$ at $a_{j}, j \neq i$, is $n_{j}$ by $\chi_{i j, m}\left(n_{1}, n_{2}\right)$ denote the first non-trivial coefficients of the expansion of $\psi_{i, m}$ at $a_{j}$, i.e.,

$$
\psi_{i, m}=\tilde{t}_{j}^{n_{i}}\left(\chi_{i j, m}+\ldots\right), \quad i \neq j
$$

Proposition 4. There exists a $\mathbb{K}$-valued potential (the $\tau$-function) defined (up to a constant) by formulas

$$
\begin{aligned}
T_{1} \tau_{m} & =\rho_{1, m} \tau_{m} \\
T_{2} \tau_{m} & =\rho_{2, m} \tau_{m} \\
\tau_{m+1} & =(-1)^{n_{1}+n_{2}} Q_{12, m} \tau_{m}
\end{aligned}
$$


Proof. The first terms in expansion of equations (4) and (7) at $a_{1}$ give

$$
\begin{aligned}
T_{2} \rho_{1, m}-\rho_{1, m} & =\left(T_{2} Q_{12, m}\right) \chi_{21, m} \\
0-\chi_{21, m} & =\left(T_{1} Q_{21, m}\right) \rho_{1}
\end{aligned}
$$

which combined together give

$$
T_{2} \rho_{1, m}=\left(1-\left(T_{2} Q_{12, m}\right)\left(T_{1} Q_{21, m}\right)\right) \rho_{1, m} .
$$

Similarly, but changing the expansion point to $a_{2}$ we obtain

$$
T_{1} \rho_{2, m}=\left(1-\left(T_{2} Q_{12, m}\right)\left(T_{1} Q_{21, m}\right)\right) \rho_{2, m} .
$$

Both equations imply

$$
\left(T_{2} \rho_{1, m}\right) \rho_{2, m}=\left(T_{1} \rho_{2, m}\right) \rho_{1, m},
$$

which is the compatibility condition of equations (10) and (11).

Expansion of equation (5) at $a_{1}$ gives

$$
\rho_{1, m+1}=-\frac{T_{1} Q_{12, m}}{Q_{12, m}} \rho_{1, m}
$$

which is the compatibility condition of equations (10) and (12). Finally, by comparing equations (6) and (9) we obtain

$$
Q_{21, m+1}=\frac{1}{Q_{12, m}}
$$

which, combined with the following consequence of expansion of (8) at $a_{2}$

$$
\rho_{2, m-1}=-\frac{T_{2} Q_{21, m}}{Q_{21, m}} \rho_{2, m}
$$

gives the compatibility condition of equations (11) and (12).

Corollary 5. Equation (13) written in terms of the $\tau$-function reads

$$
\tau_{m} T_{1} T_{2} \tau_{m}=T_{1} \tau_{m} T_{2} \tau_{m}-T_{1} \tau_{m-1} T_{2} \tau_{m+1}
$$

which is the Hirota equation 18/.

Corollary 6. Compatibility condition of equations (13) and (14) written in terms of the function $Q_{12, m}$ reads

$$
\frac{T_{1} T_{2} Q_{12, m}}{T_{2} Q_{12, m}}-\frac{T_{1} Q_{12, m}}{Q_{12, m}}=\frac{T_{1} T_{2} Q_{12, m}}{T_{1} Q_{12, m-1}}-\frac{T_{2} Q_{12, m+1}}{Q_{12, m}} .
$$




\section{Construction of solutions using vacuum functions}

Results of this Section were motivated by papers on the fundamental transformation of quadrilateral lattices in a vectorial formulation [26, 15, 25] and on the algebrogeometric interpretation of this transformation [11, 12].

In the case $N=0$ let us add superscript 0 to all functions defined above and call them the vacuum functions. The functions for arbitrary $N$ can be constructed with the help of $N$ new functions, which we define below.

Definition 2. Fix local parameters $t_{\alpha}$ at $c_{\alpha}, \alpha=1, \ldots, N$. For any $\alpha$ define the function $\phi_{\alpha, m}^{0}$ by the following conditions:

1. it has pole of the order at most $n_{1}+m$ at $b_{1}$ and the pole of order at most $n_{2}-m$ at $b_{2}$,

2 it has zeros of order at least $n_{1}$ at $a_{1}$, and of order at least $n_{2}$ at $a_{2}$,

3. it has at most simple pole at the point $c_{\alpha}$ and the first nontrivial coefficient of the expansion in $t_{\alpha}$ at $c_{\alpha}$ is normalized to one,

4. it has zero at least of the first order at the infinity point $h_{\infty}$,

5. it has at most simple poles at points $f_{\gamma}, \gamma=1, \ldots, g$.

Remark. The function $\phi_{\alpha, m}^{0}$ is unique but usually it is not $\mathbb{K}$-rational.

Lemma 7. Denote by $\psi_{i, m}^{0}(\boldsymbol{d}, \boldsymbol{e}), i=1,2$, the column with $N$ entries of the form

$$
\left[\psi_{i, m}^{0}(\boldsymbol{d}, \boldsymbol{e})\right]_{\beta}=\psi_{i, m}^{0}\left(d_{\beta}\right)-\psi_{i, m}^{0}\left(e_{\beta}\right), \quad \beta=1, \ldots, N
$$

denote by $\phi_{\boldsymbol{A}, m}^{0}$ the row with $N$ entries

$$
\left[\phi_{\boldsymbol{A}, m}^{0}\right]_{\alpha}=\phi_{\alpha, m}^{0}, \quad \alpha=1, \ldots, N
$$

and denote by $\phi_{\boldsymbol{A}, m}^{0}(\boldsymbol{d}, \boldsymbol{e})$ the $N \times N$ matrix whose element in row $\alpha$ and column $\beta$ is

$$
\left[\phi_{\boldsymbol{A}, m}^{0}(\boldsymbol{d}, \boldsymbol{e})\right]_{\alpha \beta}=\phi_{\alpha, m}^{0}\left(d_{\beta}\right)-\phi_{\alpha, m}^{0}\left(e_{\beta}\right), \quad \alpha, \beta=1, \ldots, N .
$$

Then the wave functions $\psi_{i, m}$ read

$$
\psi_{i, m}=\psi_{i, m}^{0}-\phi_{\boldsymbol{A}, m}^{0}\left[\phi_{\boldsymbol{A}, m}^{0}(\boldsymbol{d}, \boldsymbol{e})\right]^{-1} \psi_{i, m}^{0}(\boldsymbol{d}, \boldsymbol{e}) .
$$

Proof. Denote the right hand side of equation (18) by $\widehat{\psi_{i, m}^{0}}$. By building the column $\widehat{\psi_{i, m}^{0}}(\boldsymbol{d}, \boldsymbol{e})$ with $N$ entries of the form $\widehat{\psi_{i, m}^{0}}\left(d_{\beta}\right)-\widehat{\psi_{i, m}^{0}}\left(e_{\beta}\right)$ we can easily show that $\widehat{\psi_{i, m}^{0}}(\boldsymbol{d}, \boldsymbol{e})=0$. This demonstrates that the function $\widehat{\psi_{i, m}^{0}}$ satisfies constraints (3). One can check check that $\widehat{\psi_{i, m}^{0}}$ satisfies also other properties which define uniquely the function $\psi_{i, m}$. 
In the generic case denote by $H_{i, \alpha, m}^{0}$ the first nontrivial coefficient of expansion of the function $\phi_{\alpha, m}^{0}$ in the uniformization parameter $t_{i}$ at $b_{i}$, for example,

$$
\phi_{\alpha, m}^{0}=\frac{1}{t_{1}^{n_{1}+m}}\left(H_{1, \alpha, m}^{0}+\ldots\right) .
$$

Lemma 8. The corresponding expressions for $Q_{i j, m}, i \neq j$, and for $\rho_{i, m}$ read

$$
\begin{aligned}
Q_{i j, m} & =Q_{i j, m}^{0}-H_{j, \boldsymbol{A}, m}^{0}\left[\phi_{\boldsymbol{A}, m}^{0}(\boldsymbol{d}, \boldsymbol{e})\right]^{-1} \psi_{i, m}^{0}(\boldsymbol{d}, \boldsymbol{e}), \quad i \neq j, \\
\rho_{i, m} & =\rho_{i, m}^{0}\left(1+\left(T_{i} H_{i, \boldsymbol{A}, m}^{0}\right)\left[\phi_{\boldsymbol{A}, m}^{0}(\boldsymbol{d}, \boldsymbol{e})\right]^{-1} \psi_{i, m}^{0}(\boldsymbol{d}, \boldsymbol{e})\right),
\end{aligned}
$$

where $H_{i, \boldsymbol{A}, m}^{0}$ is the row with $N$ entries $H_{i, \alpha, m}^{0}$.

Proof. Because equation (19) can be obtained by expansion of formula (18) at $b_{j}$, only equation (20) needs an explanation. Notice first equation

$$
T_{i} \phi_{\alpha, m}^{0}-\phi_{\alpha, m}^{0}=\left(T_{i} H_{i, \alpha, m}^{0}\right) \psi_{i, m}^{0},
$$

which can be shown in the same way like equations of Proposition 3. Denote by $F_{i, \alpha, m}^{0}$ the first nontrivial coefficient of expansion of $\phi_{\alpha, m}^{0}$ at $a_{i}$, for example

$$
\phi_{\alpha, m}^{0}=\frac{1}{\tilde{t}_{1}^{n_{1}}}\left(F_{1, \alpha, m}^{0}+\ldots\right) \text {. }
$$

Expansion of equation (18) at $a_{i}$ gives

$$
\rho_{i, m}^{0}\left(T_{i} H_{i, \alpha, m}^{0}\right)=-F_{i, \alpha, m}^{0},
$$

which concludes the proof.

We will use the following result, which can be proven by induction with respect to the dimension of the vector space $\mathbb{V}$.

Lemma 9. Given $\boldsymbol{u} \in \mathbb{V}$ and $\boldsymbol{v}^{*} \in \mathbb{V}^{*}$, if $1_{\mathbb{V}}$ is the identity operator on $\mathbb{V}$ then

$$
\operatorname{det}\left(1_{\mathbb{V}}+\boldsymbol{u} \otimes \boldsymbol{v}^{*}\right)=1+\left\langle\boldsymbol{v}^{*}, \boldsymbol{u}\right\rangle .
$$

Proposition 10. Using the above notation the $\tau$-function can be constructed by the following formula

$$
\tau_{m}=\tau_{m}^{0} \operatorname{det} \phi_{\boldsymbol{A}, m}^{0}(\boldsymbol{d}, \boldsymbol{e}) .
$$

Proof. Notice that equation (21) implies

$$
\left[\phi_{\boldsymbol{A}, m}^{0}(\boldsymbol{d}, \boldsymbol{e})\right]^{-1} T_{i} \phi_{\boldsymbol{A}, m}^{0}(\boldsymbol{d}, \boldsymbol{e})=1_{\overline{\mathbb{K}}^{N}}+\left(\left[\phi_{\boldsymbol{A}, m}^{0}(\boldsymbol{d}, \boldsymbol{e})\right]^{-1} \psi_{i, m}^{0}(\boldsymbol{d}, \boldsymbol{e})\right) \otimes\left(T_{i} H_{i, \boldsymbol{A}, m}^{0}\right),
$$

which gives, by Lemma 9 ,

$$
\frac{\operatorname{det} T_{i} \phi_{\boldsymbol{A}, m}^{0}(\boldsymbol{d}, \boldsymbol{e})}{\operatorname{det} \phi_{\boldsymbol{A}, m}^{0}(\boldsymbol{d}, \boldsymbol{e})}=1+\left(T_{i} H_{i, \boldsymbol{A}, m}^{0}\right)\left[\phi_{\boldsymbol{A}, m}^{0}(\boldsymbol{d}, \boldsymbol{e})\right]^{-1} \psi_{i, m}^{0}(\boldsymbol{d}, \boldsymbol{e}) .
$$


Comparing equation (23) with equation (20) and taking into account equations (10)(11) we obtain

$$
\frac{\operatorname{det} T_{i} \phi_{\boldsymbol{A}, m}^{0}(\boldsymbol{d}, \boldsymbol{e})}{\operatorname{det} \phi_{\boldsymbol{A}, m}^{0}(\boldsymbol{d}, \boldsymbol{e})}=\frac{T_{i} \tau_{m} / T_{i} \tau_{m}^{0}}{\tau_{m} / \tau_{m}^{0}} .
$$

Notice the following equation

$$
\phi_{\alpha, m+1}^{0}-\phi_{\alpha, m}^{0}=-\frac{H_{2, \alpha, m}^{0}}{Q_{12, m}^{0}} \psi_{1, m}^{0},
$$

which can be shown in the same way like equations of Proposition 3. It implies that

$$
\left[\phi_{\boldsymbol{A}, m}^{0}(\boldsymbol{d}, \boldsymbol{e})\right]^{-1} \phi_{\boldsymbol{A}, m+1}^{0}(\boldsymbol{d}, \boldsymbol{e})=1_{\overline{\mathbb{K}}^{N}}-\frac{1}{Q_{12, m}^{0}}\left(\left[\phi_{\boldsymbol{A}, m}^{0}(\boldsymbol{d}, \boldsymbol{e})\right]^{-1} \psi_{1, m}^{0}(\boldsymbol{d}, \boldsymbol{e})\right) \otimes\left(H_{2, \boldsymbol{A}, m}^{0}\right),
$$

which gives

$$
\frac{\operatorname{det} \phi_{\boldsymbol{A}, m+1}^{0}(\boldsymbol{d}, \boldsymbol{e})}{\operatorname{det} \phi_{\boldsymbol{A}, m}^{0}(\boldsymbol{d}, \boldsymbol{e})}=1-\frac{1}{Q_{12, m}^{0}} H_{2, \boldsymbol{A}, m}^{0}\left[\phi_{\boldsymbol{A}, m}^{0}(\boldsymbol{d}, \boldsymbol{e})\right]^{-1} \psi_{1, m}^{0}(\boldsymbol{d}, \boldsymbol{e}) .
$$

Comparing equation (25) with equation (19) and taking into account equation (12) we obtain

$$
\frac{\operatorname{det} \phi_{\boldsymbol{A}, m+1}^{0}(\boldsymbol{d}, \boldsymbol{e})}{\operatorname{det} \phi_{\boldsymbol{A}, m}^{0}(\boldsymbol{d}, \boldsymbol{e})}=\frac{\tau_{m+1} / \tau_{m+1}^{0}}{\tau_{m} / \tau_{m}^{0}}
$$

which, together with equation (24), concludes the proof.

Corollary 11. Notice that equation (22) is valid up to a (nonessential) change of the initial value of the $\tau$-function, which is due to introduction of the integration constant from formulas (24) and (26).

Corollary 12. Starting with $\mathbb{K}$-valued function $\tau_{m}^{0}$ and the local parameters $t_{\alpha}$ at $c_{\alpha}$ chosen in a consistent way with the action of the Galois group $G(\overline{\mathbb{K}} / \mathbb{K})$ on $\mathcal{C}_{\overline{\mathbb{K}}}$ we obtain $\mathbb{K}$-valued function $\tau_{m}$.

\section{Multisoliton solutions}

We present here explicit formulas for the vacuum functions in the simplest case $g=$ 0 . Then with the help of these expressions we present some examples of $N$-soliton solutions. In constructing the vacuum functions we will use the standard parameter $t$ on the projective line $\mathbb{P}(\mathbb{K})$ and we put $h_{\infty}=\infty$.

Explicit forms of the wave functions read

$$
\begin{aligned}
& \psi_{1, m}^{0}=\frac{1}{\left(t-b_{1}\right)^{n_{1}+1+m}} \frac{\left(t-a_{1}\right)^{n_{1}}\left(t-a_{2}\right)^{n_{2}}\left(b_{1}-b_{2}\right)^{n_{2}-m}}{\left(b_{1}-a_{1}\right)^{n_{1}}\left(b_{1}-a_{2}\right)^{n_{2}}\left(t-b_{2}\right)^{n_{2}-m}}, \\
& \psi_{2, m}^{0}=\frac{1}{\left(t-b_{2}\right)^{n_{2}+1-m}} \frac{\left(b_{2}-b_{1}\right)^{n_{1}+m}\left(t-a_{1}\right)^{n_{1}}\left(t-a_{2}\right)^{n_{2}}}{\left(t-b_{1}\right)^{n_{1}+m}\left(b_{2}-a_{1}\right)^{n_{1}}\left(b_{2}-a_{2}\right)^{n_{2}}},
\end{aligned}
$$


which gives formulas for the functions $Q_{12, m}^{0}$ and $Q_{21, m}^{0}$

$$
\begin{aligned}
Q_{12, m}^{0} & =\frac{(-1)^{n_{2}-m}}{\left(b_{2}-b_{1}\right)^{n_{1}-n_{2}+1+2 m}} \frac{\left(b_{2}-a_{1}\right)^{n_{1}}\left(b_{2}-a_{2}\right)^{n_{2}}}{\left(b_{1}-a_{1}\right)^{n_{1}}\left(b_{1}-a_{2}\right)^{n_{2}}}, \\
Q_{21, m}^{0} & =\frac{(-1)^{n_{1}+m}}{\left(b_{1}-b_{2}\right)^{n_{2}-n_{1}+1-2 m}} \frac{\left(b_{1}-a_{1}\right)^{n_{1}}\left(b_{1}-a_{2}\right)^{n_{2}}}{\left(b_{2}-a_{1}\right)^{n_{1}}\left(b_{2}-a_{2}\right)^{n_{2}}},
\end{aligned}
$$

and for the functions $\rho_{1, m}$ and $\rho_{2, m}$

$$
\begin{aligned}
& \rho_{1, m}^{0}=\frac{(-1)^{n_{1}}}{\left(a_{1}-b_{1}\right)^{2 n_{1}+1+m}} \frac{\left(a_{1}-a_{2}\right)^{n_{2}}\left(b_{1}-b_{2}\right)^{n_{2}-m}}{\left(b_{1}-a_{2}\right)^{n_{2}}\left(a_{1}-b_{2}\right)^{n_{2}-m}}, \\
& \rho_{2, m}^{0}=\frac{(-1)^{n_{2}}}{\left(a_{2}-b_{2}\right)^{2 n_{2}+1-m}} \frac{\left(b_{2}-b_{1}\right)^{n_{1}+m}\left(a_{2}-a_{1}\right)^{n_{1}}}{\left(a_{2}-b_{1}\right)^{n_{1}+m}\left(b_{2}-a_{1}\right)^{n_{1}}} .
\end{aligned}
$$

Explicit form of the vacuum $\tau$-function reads

$$
\tau_{m}^{0}=\frac{(-1)^{\left[n_{1}\left(n_{1}-1\right)+n_{2}\left(n_{2}-1\right)+m(m+1)\right] / 2}}{\left(a_{1}-b_{1}\right)^{n_{1}\left(n_{1}+m\right)}\left(a_{2}-b_{2}\right)^{n_{2}\left(n_{2}-m\right)}} \frac{\left(a_{1}-a_{2}\right)^{n_{1} n_{2}}\left(b_{1}-b_{2}\right)^{\left(n_{2}-m\right)\left(m+n_{1}\right)}}{\left(b_{1}-a_{2}\right)^{n_{2}\left(n_{1}+m\right)}\left(a_{1}-b_{2}\right)^{n_{1}\left(n_{2}-m\right)}} .
$$

The functions $\phi_{\alpha, m}^{0}, \alpha=1, \ldots, N$ have the form

$$
\phi_{\alpha, m}^{0}=\frac{1}{t-c_{\alpha}} \frac{\left(t-a_{1}\right)^{n_{1}}\left(t-a_{2}\right)^{n_{2}}\left(c_{\alpha}-b_{1}\right)^{n_{1}+m}\left(c_{\alpha}-b_{2}\right)^{n_{2}-m}}{\left(c_{\alpha}-a_{1}\right)^{n_{1}}\left(c_{\alpha}-a_{2}\right)^{n_{2}}\left(t-b_{1}\right)^{n_{1}+m}\left(t-b_{2}\right)^{n_{2}-m}}
$$

and can be used, due to Proposition 10, to construct the $\tau$-function for arbitrary $N$.

Let $\mathbb{L}=\mathbb{F}_{q^{\ell}} \subset \overline{\mathbb{K}}$ be a field of rationality of all the points used in the construction. Recall [23] that if $q=p^{k}$ then the Galois group $G(\mathbb{L} / \mathbb{K})$ is the cyclic group of order $\ell$ and is generated by $\sigma_{F}^{k}$, where $\sigma_{F}$ is the Frobenius automorphism of $\mathbb{L}$ defined as $\sigma_{F}(a)=a^{p}$. Therefore possible systems of points $c_{\alpha}$ and pairs $\left\{d_{\beta}, e_{\beta}\right\}$ can be grouped into $\mathbb{K}$-rational clusters (orbits of the group $G(\mathbb{L} / \mathbb{K})$ ) of the lengths being divisors of $\ell$. In the standard nomenclature the clusters of length one correspond to solitons, and clusters of length two give rise to breathers (the position of poles $c_{\alpha}$ of the wave functions must be symmetric with respect to the complex conjugation). In finite fields we encounter new types of solutions (without direct analogs in the complex field case) which come from clusters of lengths greater then two. The analog of the breather solution will be presented in Example 1. Let us call the $N$-soliton solution of order $\ell$ the $\mathbb{K}$-rational $N$-soliton solution with parameters in extension of $\mathbb{K}$ of order $\ell$. In this terminology the standard $N$-soliton solutions are of order one, while the breather solution is a 2-soliton solution of order two. A 3 -soliton solution of order three and a 2 -soliton solution of order four are presented in Examples 2 and 3. We remark that the above terminology is not completely distinctive.

Notice that the variables $n_{1}, n_{2}$ and $m$ enter exponentially in the functions $\tau_{m}^{0}$ and $\phi_{\alpha, m}^{0}$ This implies that the $\tau$-function is periodic in $n_{1}, n_{2}$ and $m$ with the periods being divisors of $q^{\ell}-1$, which is the order of the cyclic multiplicative group $\mathbb{L}_{*}$. 
Finally, we present the examples. For any example we describe the fields $\mathbb{K}$ and $\mathbb{L}$ giving first the numbers $q=p^{k}$ and $\ell$ and then writing down the polynomial $w(x)$ over $\mathbb{F}_{p}$ used to construct multiplication in the field $\mathbb{L}$. We represent elements of $\mathbb{L}$ as elements of the vector space $\mathbb{F}_{p}^{k l}$. Then we give the points used in the construction of the solution of the Hirota equation presenting also the action of the Galois group $G(\mathbb{L} / \mathbb{K})$ on them.

Example 1. A breather solution of the Hirota equation in $\mathbb{F}_{5}$. Parameters of the solution take values in extension $\mathbb{F}_{5^{2}}$ of $\mathbb{F}_{5}$ by the polynomial $w(x)=x^{2}+x+1$. The corresponding Galois group reads $G\left(\mathbb{F}_{5^{2}} / \mathbb{F}_{5}\right)=\{i d, \sigma\}$, where $\sigma^{2}=i d$. The parameters of the solution are chosen as follows

$$
\begin{aligned}
& a_{1}=(00), a_{2}=(02), b_{1}=(01), b_{2}=(04) \\
& c_{1}=(10), c_{2}=\sigma\left(c_{1}\right)=(44) \\
& d_{1}=(11), d_{2}=\sigma\left(d_{1}\right)=(40) \\
& e_{1}=(13), e_{2}=\sigma\left(e_{1}\right)=(42)
\end{aligned}
$$

This solution is presented in Figures 1 and 2. The elements of $\mathbb{F}_{5}$ are represented by: $\square_{-}(00), \square_{-}(01), \square_{-}(02), \square_{-}(03), \square_{-}(04)$.

The periods in variables $n_{1}, n_{2}$ and $m$ are 12, 24 and 24, correspondingly. Notice that figure for $m=5$ can be obtained from the figure for $m=1$ by a shift in $n_{2}$ by four.

Example 2. A 3 -soliton solution of order 3 of the Hirota equation in $\mathbb{F}_{5}$. This solution is the first one in the generalized breather class, a new type not present in the standard complex field case. Parameters of the solution take values in extension $\mathbb{F}_{5^{3}}$ of $\mathbb{F}_{5}$ by the polynomial $w(x)=x^{3}+x^{2}+1$. The corresponding Galois group reads $G\left(\mathbb{F}_{5^{3}} / \mathbb{F}_{5}\right)=\left\{i d, \sigma, \sigma^{2}\right\}$, where $\sigma^{3}=i d$. The parameters of the solution are chosen as follows:

$$
\begin{aligned}
& a_{1}=(004), a_{2}=(003), b_{1}=(002), b_{2}=(001) \\
& c_{1}=(022), c_{2}=\sigma\left(c_{1}\right)=(120), c_{3}=\sigma^{2}\left(c_{1}\right)=(412), \\
& d_{1}=(020), d_{2}=\sigma\left(d_{1}\right)=(123), d_{3}=\sigma^{2}\left(d_{1}\right)=(410), \\
& e_{1}=(010), e_{2}=\sigma\left(e_{1}\right)=(314), e_{3}=\sigma^{2}\left(e_{1}\right)=(230) .
\end{aligned}
$$

This solution is presented in Figures 3 and 4 . The elements of $\mathbb{F}_{5}$ are represented like in Example 1. From the figures one can deduce that the periods in all variables $n_{1}, n_{2}$ and $m$ are maximal and equal to 124 .

Example 3. The goal of this example is to present a solution in a "small" field $\mathbb{F}_{4}$ obtained from parameters taking values in a relatively bigger field $\mathbb{F}_{256}$. The field $\mathbb{F}_{256}$ is chosen as extension of $\mathbb{F}_{2}$ by the polynomial $w(x)=x^{8}+x^{6}+x^{5}+x+1$. The elements (00000000), (00000001), (11110000) and (11110001) of $\mathbb{F}_{256}=\mathbb{F}_{2^{8}}$ form a subfield isomorphic to $\mathbb{F}_{4}$. The Galois group is generated by $\sigma=\sigma_{F}^{2}$, and reads $G\left(\mathbb{F}_{256} / \mathbb{F}_{4}\right)=\left\{i d, \sigma, \sigma^{2}, \sigma^{3}\right\}$, where $\sigma^{4}=i d$. The parameters of the solution are as follows:

$$
a_{1}=(00000000), a_{2}=(00000001), b_{1}=(11110000), b_{2}=(11110001)
$$




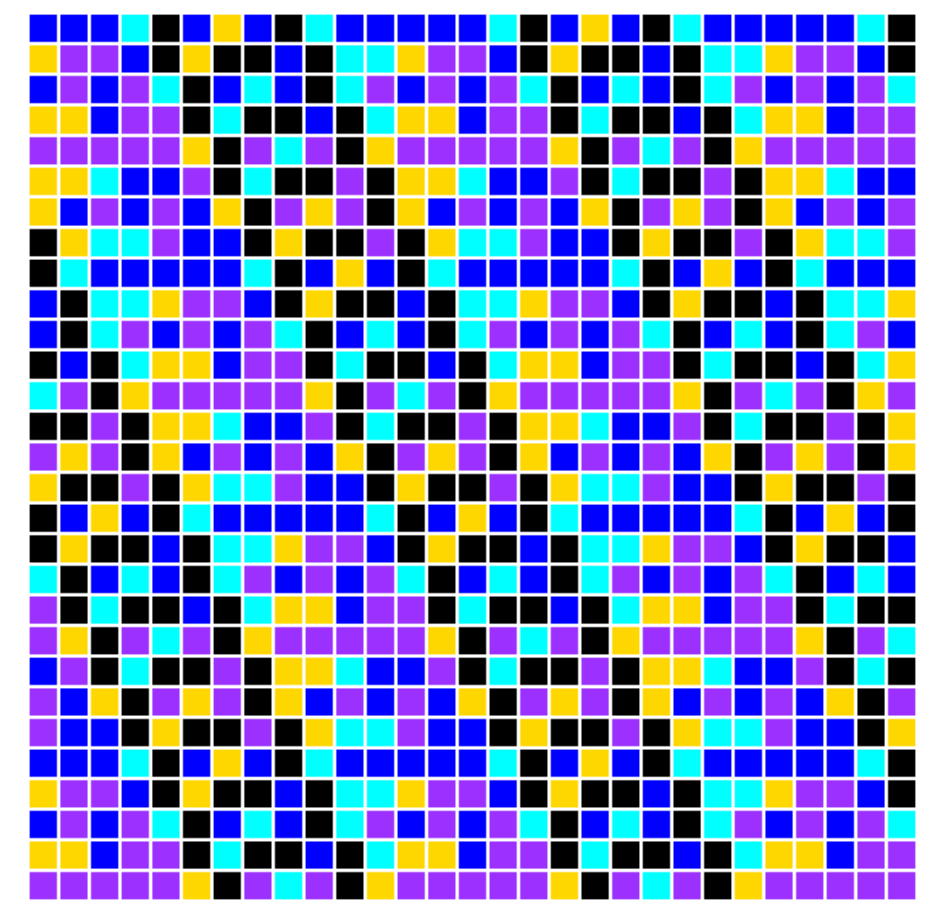

Figure 1: A breather solution of the Hirota equation in $\mathbb{F}_{5}$ described in Example $\mathbb{1}$; $n_{1}$ range from 0 to 28 (directed to the right), $n_{2}$ range from 0 to 28 (directed up), $m=1$.

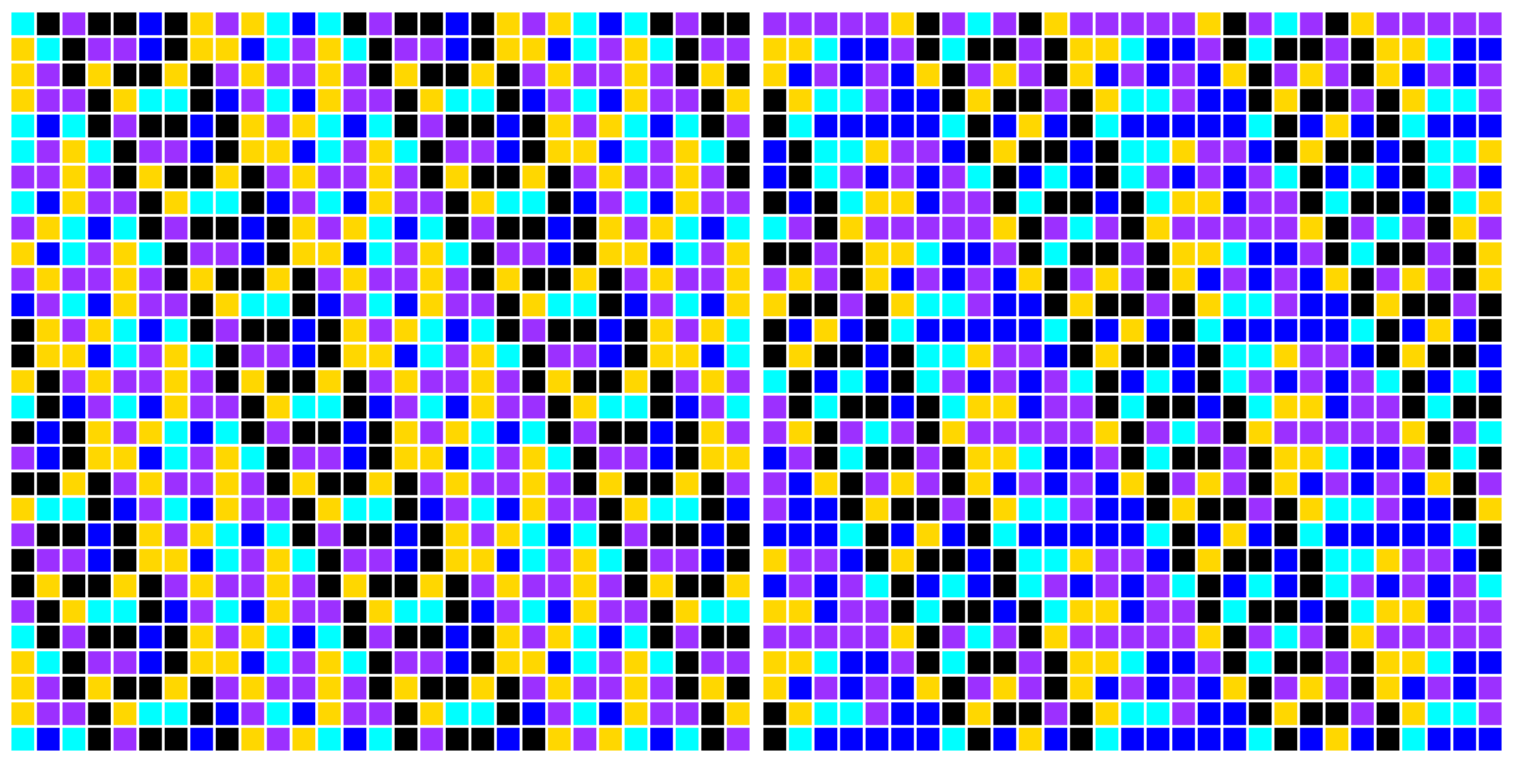

Figure 2: A breather solution as in Figure 1 1 for $m=2$ and $m=5$. 

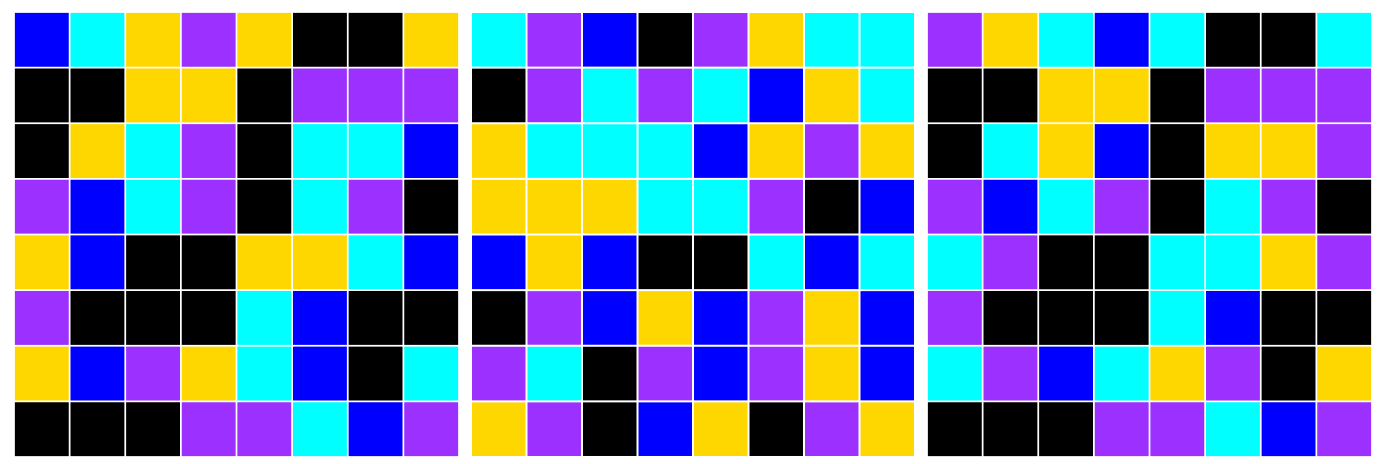

Figure 3: A breather of order 3 solution of the Hirota equation in $\mathbb{F}_{5}$ described in Example 2 2; $n_{1}$ range from 0 to 8 (directed to the right), $n_{2}$ range from 0 to 8 (directed up), $m=0,4$ and 62 .

$$
\begin{aligned}
& c_{1}=(00010010), c_{2}=\sigma\left(c_{1}\right)=(11100011), \\
& d_{1}=(00001010), d_{2}=\sigma\left(d_{1}\right)=(00001001), \\
& e_{1}=\sigma^{2}\left(d_{1}\right)=(00011000), e_{2}=\sigma^{3}\left(d_{1}\right)=(11101010) .
\end{aligned}
$$

Here the points $c_{1}$ and $c_{2}$ are chosen from a subfield of $\mathbb{F}_{256}$ isomorphic to $\mathbb{F}_{16}$ and form a cluster of length two. The points $c_{1}, c_{2}, d_{1}$ and $d_{2}$ form a cluster of length four in a way compatible with the $\mathbb{F}_{4}$-rationality condition (2). We obtain therefore a 2-soliton solution of order four, which also has no direct counterpart in the complex field case.

The solution is presented in Figure 5 for $m=0$; The elements of $\mathbb{F}_{4}$ are represented by: $\mathbf{\square}-(00000000), \mathbf{\square}-(00000001), \mathbf{\square}-(11110000), \square_{-}(11110001)$. The period in all variables is the same and equals 51 .

\section{Conclusion and remarks}

In the paper, motivated by recent developments of integrable discrete geometry, we presented algebro-geometric method of construction of solutions of the Hirota equation over finite fields. It turns out that the main ideas used for integrable systems over the field of complex numbers, e.g., application of the Riemann-Roch theorem, can be transferred to the level of finite fields without essential modifications. We would like to stress that although finite fields consist of finite number of elements the corresponding algebraic curves have infinite number of points (taking into account the algebraic completion of the field) which gives rise to infinite families of solutions of the equation.

We have presented examples of pure (for the simplest algebraic curve being the projective line) multisoliton solutions of the Hirota equation. Less trivial examples which use techniques on Jacobians of algebraic curves will be the subject of another paper [4. 


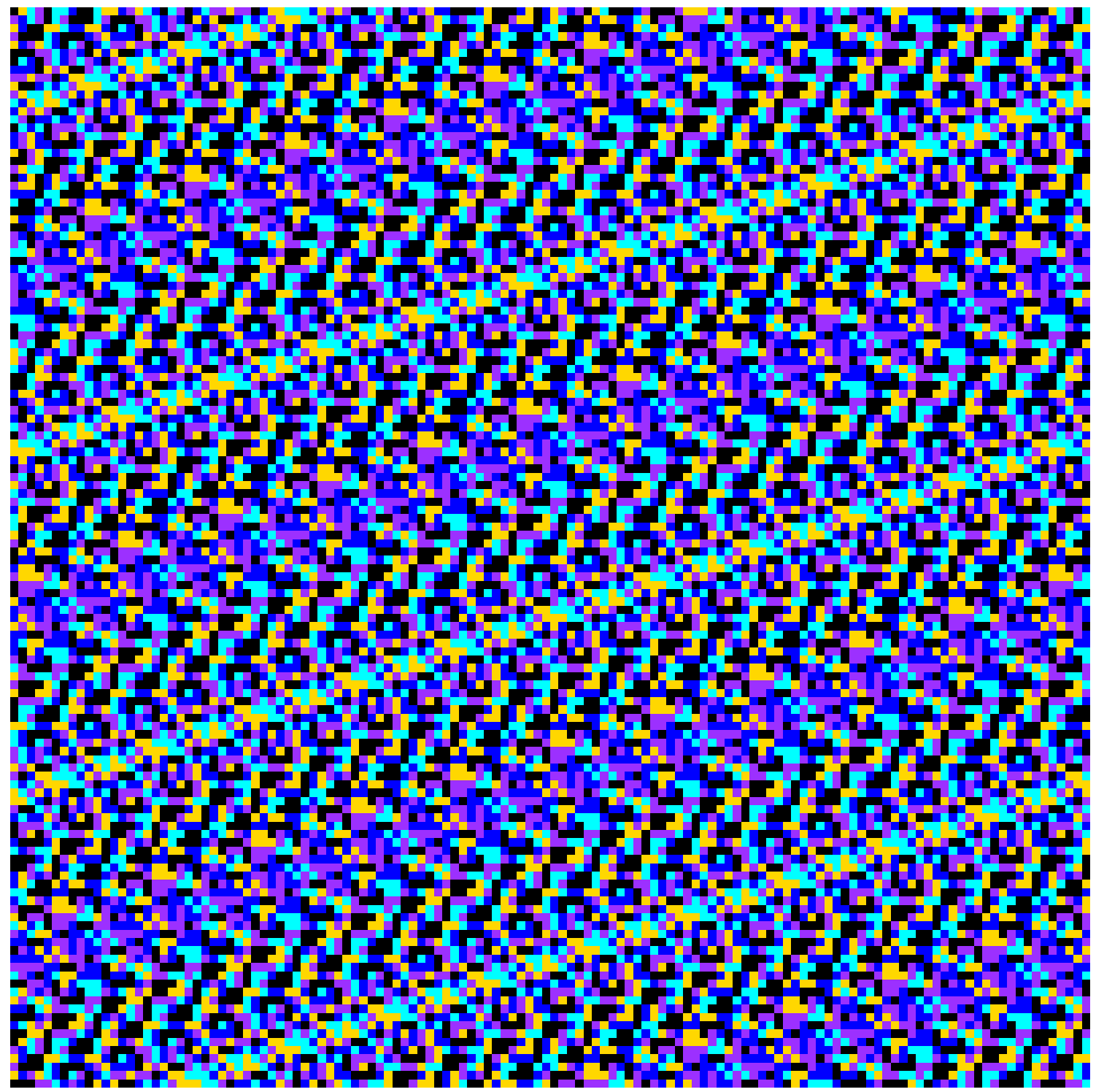

Figure 4: A "global" view of the first picture $(m=0)$ of the generalized breather solution presented in Figure 3; $n_{1}$ and $n_{2}$ range from 0 to 129. 
口n grang a

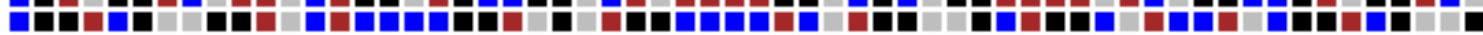
(10

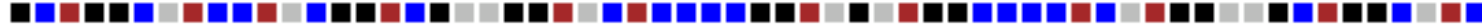
Hat

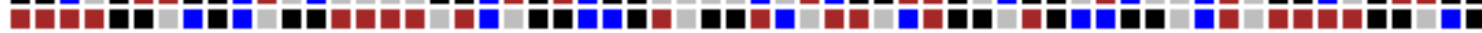

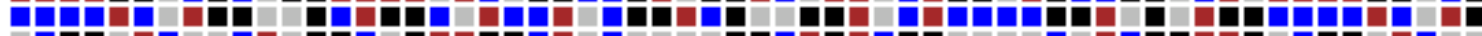

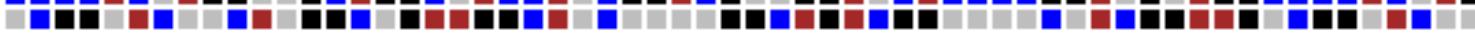

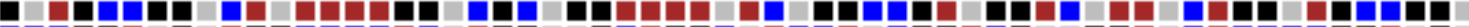

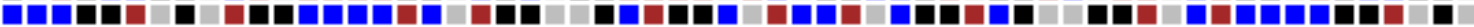
Q

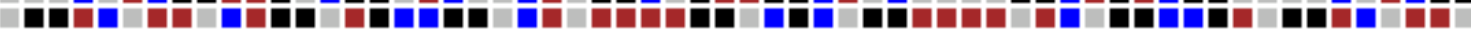
a

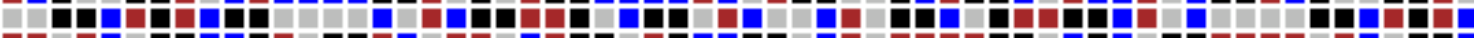
a Gna

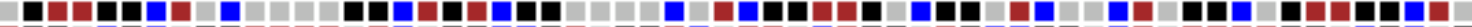
R n

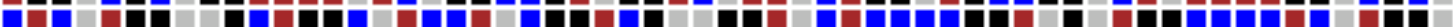

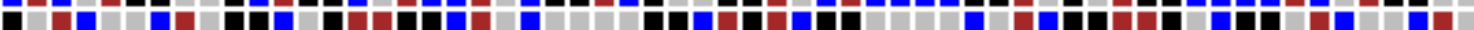

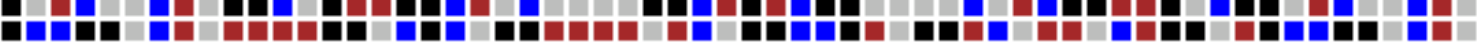

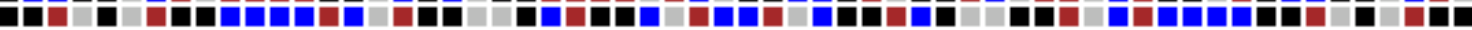

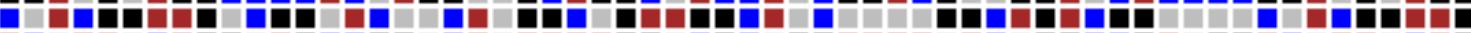
(1)

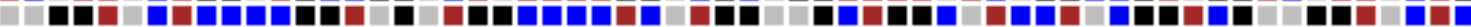
ang HAt

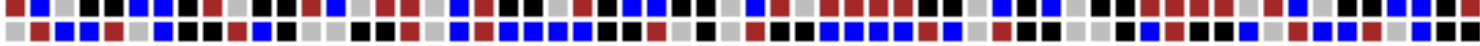
ang

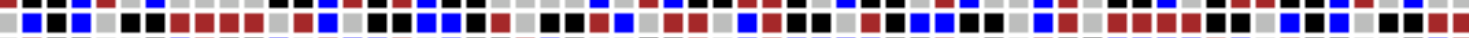

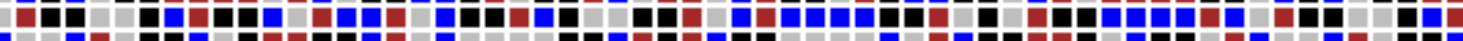

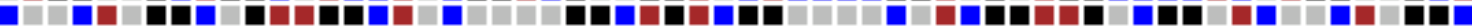
rn

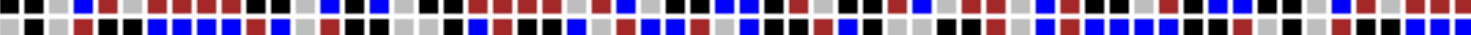

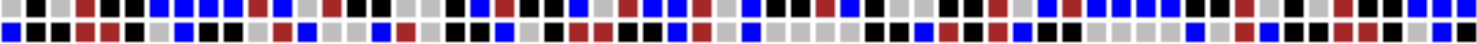

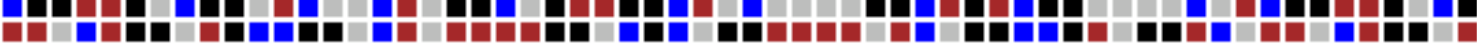

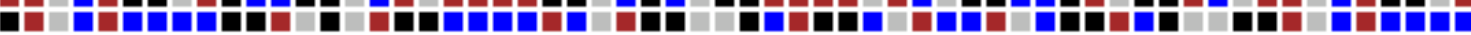
Qqu

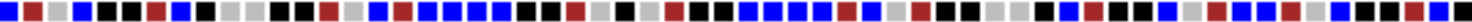
H

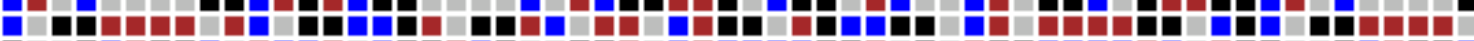

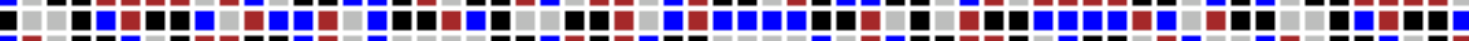

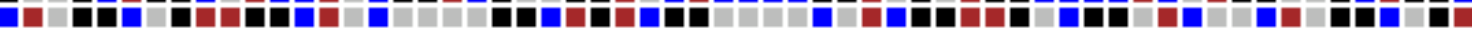

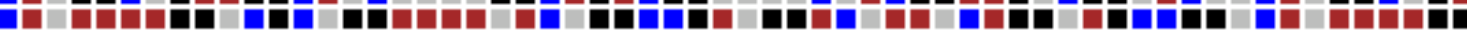

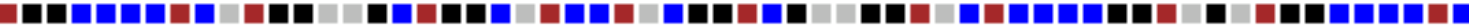
19

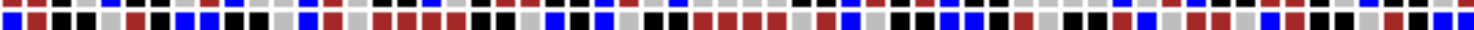

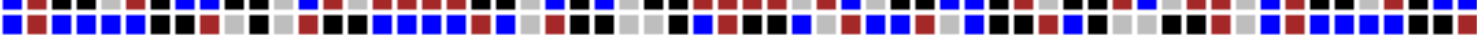

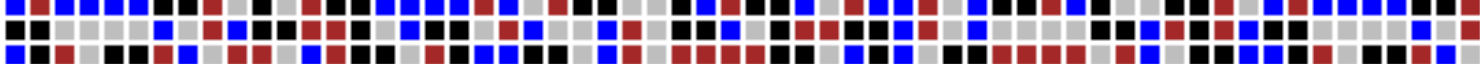

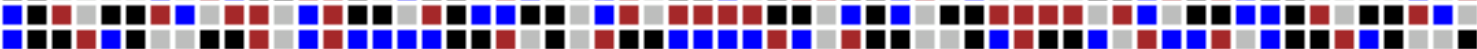

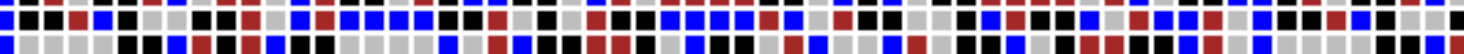

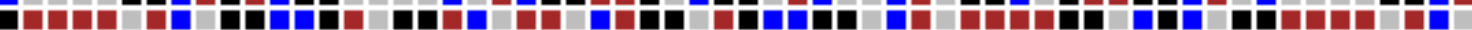

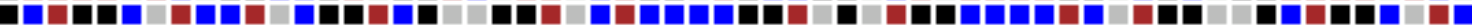

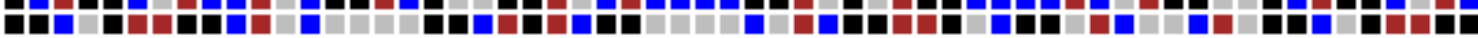

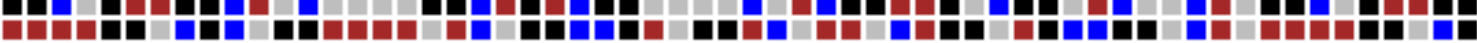
atate

Figure 5: A 2-soliton solution of order four of the Hirota equation in $\mathbb{F}_{4} ; n_{1}$ and $n_{2}$ range from 0 to $59, m=0$. 


\section{Acknowledgments}

The paper was partially supported by KBN grant no. 2P03B12622.

\section{References}

[1] A. A. Akhmetshin, I. M. Krichever, and Y. S. Volvovski, Discrete analogs of the Darboux-Egoroff metrics, hep-th/9905168.

[2] G. W. Anderson, Rank one elliptic A-modules and A-harmonic series, Duke Math. J. 73 (1994), 491-542.

[3] E. D. Belokolos, A. I. Bobenko, V. Z. Enol'skii, A. R. Its, and V. B. Matveev, Algebro-geometric approach to nonlinear integrable equations, Springer-Verlag, Berlin, 1994.

[4] M. Białecki and A. Doliwa, in preparation.

[5] A. Bobenko, M. Bordemann, Ch. Gunn, and U. Pinkall, On two integrable cellular automata, Comm. Math. Phys. 158 (1993), 127-134.

[6] M. Bruschi and P. M. Santini, Cellular automata in 1+1, 2+1 and $3+1$ dimensions, constants of motion and coherent structures, Physica D 70 (1994), 185-209.

[7] A. Doliwa, Geometric discretisation of the Toda system, Phys. Lett. A 234 (1997), 187-192.

[8] _ Discrete integrable geometry with ruler and compass, Symmetries and Integrability of Difference Equations (P. Clarkson and F. Nijhoff, eds.), Cambridge University Press, 1999, pp. 122-136.

[9] _ Quadratic reductions of quadrilateral lattices, J. Geom. Phys. 30 (1999), 169-186.

[10] L Lattice geometry of the Hirota equation, SIDE III - Symmetries and Integrability of Difference Equations (D. Levi and O. Ragnisco, eds.), CMR Proceedings and Lecture Notes, vol. 25, AMS, Providence, 2000, pp. 93-100.

[11] _ The Darboux-type transformations of integrable lattices, Rep. Math. Phys. 48 (2001), 59-66.

[12] _ Integrable multidimensional discrete geometry: Quadrilateral lattices, their transformations and reductions, Integrable Hierarchies and Modern Physical Theories (H. Aratyn and A. S. Sorin, eds.), Kluwer, Dordrecht, 2001, pp. 355389. 
[13] A. Doliwa and P. M. Santini, Multidimensional quadrilateral lattices are integrable, Phys. Lett. A 233 (1997), 365-372.

[14] - The symmetric, D-invariant and Egorov reductions of the quadrilateral lattice, J. Geom. Phys. 36 (2000), 60-102.

[15] A. Doliwa, P. M. Santini, and M. Mañas, Transformations of quadrilateral lattices, J. Math. Phys. 41 (2000), 944-990.

[16] V. Drinfeld, Commutative subrings of some noncommutative rings, Functional Anal. Appl 11 (1977), 9-12.

[17] R. Hirota, Nonlinear partial difference equations. III. Discrete sine-Gordon equation, J. Phys. Soc. Jpn. 43 (1977), 2079-2086.

[18] _ Discrete analogue of a generalized Toda equation, J. Phys. Soc. Jpn. 50 (1981), 3785-3791.

[19] J. W. P. Hirschfeld, Projective geometries over finite fields, Clarendon Press, Oxford, 1998.

[20] N. Koblitz, Algebraic aspects of cryptography, Springer, Berlin, 1998.

[21] I. M. Krichever, O. Lipan, P. Wiegmann, and A. Zabrodin, Quantum integrable models and discrete classical Hirota equations, Commun. Math. Phys. 188 (1997), 267-304.

[22] I. M. Krichever, P. Wiegmann, and A. Zabrodin, Elliptic solutions to difference non-linear equations and related many body problems, Commun. Math. Phys. 193 (1998), 373-396.

[23] S. Lang, Algebra, Addison-Wesley, Reading, Mass., 1970.

[24] R. Lidl and H. Niederreiter, Introduction to finite fields and their applications, Univ. Press, Cambridge, 1986.

[25] M. Mañas, Fundamental transformations for quadrilateral lattices: first potentials and $\tau$-functions, symmetric and pseudo-Egorov reductions, J. Phys A: Math. Gen. 34 (2001), 10413-10421.

[26] M. Mañas, A. Doliwa, and P. M. Santini, Darboux transformations for multidimensional quadrilateral lattices. I, Phys. Lett. A 232 (1997), 99-105.

[27] A. V. Mikhailov, Integrability of a two-dimensional generalization of the Toda chain, JETP Lett. 30 (1979), 414-418. 
[28] S. Moriwaki, A. Nagai, J. Satsuma, T. Tokihiro, M. Torii, D. Takahashi, and J. Matsukidaira, $2+1$ dimensional soliton cellular automaton, Symmetries and Integrability of Difference Equations (P. Clarkson and F. Nijhoff, eds.), Cambridge University Press, 1999, pp. 334-342.

[29] D. Mumford, An algebro-geometric construction of commuting operators and of solutions to the Toda lattice equation, Korteweg-de Vries equation, and related nonlinear equations, Proceedings of the International Symposium on Algebraic Geometry (M. Nagata, ed.), Kinokuniya, Tokyo, 1978, pp. 115-153.

[30] Y. Nakamura and A. Mukaihira, Dynamics of the finite Toda molecule over finite fields and a decoding algorithm, Phys. Lett. A 249 (1998), 295-302.

[31] H. Stichtenoth, Algebraic function fields and codes, Springer-Verlag, Berlin, 1993.

[32] D. S. Thakur, Integrable systems and number theory in finite characterictic, Physica D 152-153 (2001), 1-8.

[33] T. Tokihiro, D. Takahashi, J. Matsukidaira, and J. Satsuma, From soliton equations to integrable cellular automata through a limiting procedure, Phys. Rev. Lett. 76 (1996), 3247-3250.

[34] S. Wolfram, Theory and application of cellular automata, World Scientific, Singapore, 1986. 